\title{
ON ORDERED HARMONIC BOUNDED VARIATION
}

\author{
C. L. BELNA
}

Abstract. An example is given of a continuous real function that is of ordered harmonic bounded variation but not of harmonic bounded variation.

Let $f$ be a real-valued function defined on $[0,1]$, and for each open interval $I=(a, b) \subset[0,1]$ set $f(I)=f(b)-f(a)$. Then $f$ is said to be of harmonic bounded variation $(H B V)$ on $[0,1]$ if

$$
\sup \sum\left|f\left(I_{n}\right)\right| / n<\infty,
$$

where the supremum is taken over all sequences of nonoverlapping open intervals $I_{1}, I_{2}, \ldots$ in $[0,1]$; if $(\dagger)$ holds when the supremum is taken over all sequences of open intervals $I_{1}, I_{2}, \ldots$ in $[0,1]$ for which either $I_{j}<I_{j+1}$ for each index $j$ or $I_{j+1}<I_{j}$ for each index $j$ (where $I<J$ means $I$ lies to the left of $J$ ), then $f$ is said to be of ordered harmonic bounded variation $(O H B V)$ on $[0,1]$. These two function classes were introduced by $D$. Waterman in [1] and [2], respectively, and in [2] he asked whether the inclusion $H B V \subset O H B V$ is proper. Here we show that it is.

Lemma. Let $N$ be a nonnegative integer, $M$ a positive integer, and $0<\varepsilon<1$. Then there exists an integer $T>M$ and a corresponding sequence of positive numbers $A_{N+1} \geqslant A_{N+2} \geqslant \cdots \geqslant A_{N+T}$ for which the following hold:

(i) $\sum_{j=1}^{M} A_{N+j} / j<\varepsilon$,

(ii) $\sum_{j=1}^{T} A_{N+j} /(N+j)>1$ but $\sum_{j=1}^{T} A_{N+j} / j<4$,

(iii) $\sum_{j=1}^{t} A_{N+t+1-j} / j<6 \varepsilon(1 \leqslant t \leqslant T)$.

Proof. Define $A_{N+1}=A_{N+2}=\cdots=A_{N+M}=\varepsilon /(N+M+1)$ and

$$
A_{j}=A_{N+1} / k \text { for }(N+M) 2^{k-1}<j<(N+M) 2^{k}(k>1) .
$$

Noting that (i) is satisfied, we proceed to prove (ii).

First observe that if $N+T=(N+M) 2^{K}(K>1)$, then

$$
\begin{aligned}
& \sum_{j=1}^{T} A_{N+j} /(N+j) \\
& \quad=A_{N+1}\left\{\sum_{j=1}^{M} 1 /(N+j)+\sum_{k=1}^{K}\left(\frac{1}{k}\right) \sum\left((N+M) 2^{k-1},(N+M) 2^{k}\right]\right\},
\end{aligned}
$$

where we use the notation $\Sigma(a, b]=\sum_{j=a+1}^{b} 1 / j$. For $K=1$, the right-hand side of (*) is $<A_{N+1}(M+1)$, which by the definition of $A_{N+1}$ is $<1$. Also, since

Received by the editors August 1, 1979.

1980 Mathematics Subject Classification. Primary 26A45. 


$$
\ln 2>\sum(n, 2 n] \rightarrow \ln 2 \text { as } n \rightarrow \infty,
$$

the second series on the right-hand side of $(*)$ is a partial sum of a divergent series with its $k$ th term $(k>1)$ less than $(\ln 2) / 2$; hence, for some $T$ we have

$$
1<\sum_{j=1}^{T} A_{N+j} /(N+j)<2
$$

which proves the first part of (ii). The second part follows from (**) and the simple observation that

$$
\sum_{j=1}^{T} A_{N+j} / j-\sum_{j=1}^{T} A_{N+j} /(N+j)=\sum_{j=1}^{T} N A_{N+j} / j(N+j)<\sum_{j=1}^{T} \varepsilon / j(N+j)<2 .
$$

To begin consideration of (iii) we note that $A_{N+t+1-j}=A_{N+j}$ for $j<t<M$, and hence, that (iii) for $1 \leqslant t \leqslant M$ follows from (i).

Now suppose $t$ satisfies $(N+M) 2^{K-1}<N+t<(N+M) 2^{K}$ for some $K>1$. Then let $S_{n}(n=1,2, \ldots, K)$ denote the sum

$$
\sum_{j} A_{N+t+1-j} / j \text { for }(N+M) 2^{K-n}<N+t+1-j<(N+M) 2^{K-n+1},
$$

and let $S_{K+1}$ denote this sum for $N+1 \leqslant N+t+1-j<N+M$.

Consider $S_{1}$. For the indices $j$ involved in this sum, we have $A_{N+t+1-j}=$ $A_{N+1} / K$ and hence

$$
\begin{aligned}
S_{1} & \leqslant\left(A_{N+1} / K\right)\left(1+\sum\left(1,(N+M) 2^{K-1}\right]\right) \\
& <[\varepsilon / K(N+M+1)]\left[1+\ln (N+M) 2^{K-1}\right]<2 \varepsilon .
\end{aligned}
$$

Similarly $S_{2}<2 \varepsilon$.

Now consider $S_{n}$ for $3 \leqslant n \leqslant K$. Since $A_{N+t+1-j}=A_{N+1} /(K-n+1)$ for each of the $(N+M) 2^{K-n}$ indices $j$ involved in $S_{n}$ and since the first of these indices is greater than $(N+M) 2^{K-2}$, which is the number of terms in $S_{2}$, we see that

$$
\begin{aligned}
S_{n} & <\left[A_{N+1} /(K-n+1)\right]\left[(N+M) 2^{K-n} /(N+M) 2^{K-2}\right] \\
& =\left[A_{N+1} /(K-n+1)\right] / 2^{n-2} .
\end{aligned}
$$

Then, since $A_{N+1}<\varepsilon$, it follows that $S_{n}<\varepsilon / 2^{n-2}$ for $3<n \leqslant K$.

Combining these estimates with the observation that $S_{K+1}$ is dominated by $\sum_{j=1}^{M} A_{N+j} / j$ which by (i) is $<\varepsilon$, we have

$$
\sum_{j=1}^{t} A_{N+t+1-j} / j<2 \varepsilon+2 \varepsilon+\varepsilon \sum_{n=3}^{K} 2^{2-n}+\varepsilon<6 \varepsilon,
$$

and the lemma is proved.

THEOREM. There exists a continuous $f \in O H B V-H B V$.

Proof. Choose positive numbers $\varepsilon_{1}, \varepsilon_{2}, \ldots$ so that $\sum_{n=1}^{\infty} \varepsilon_{n}=1$. Then apply the Lemma for $N=0, M=M_{1} \equiv 1, \varepsilon=\varepsilon_{1}$ to obtain an integer $T_{1}>1$ and a 
sequence of positive numbers $A_{1} \geqslant A_{2} \geqslant \cdots \geqslant A_{T_{1}}$ such that

(i) $\sum_{j=1}^{M_{1}} A_{j} / j<\varepsilon_{1}$,

(ii) $1<\sum_{j=1}^{T_{1}} A_{j} / j<4$,

(iii) $\sum_{j=1}^{t} A_{t+1-j} / j<6 \varepsilon_{1}\left(1 \leqslant t<T_{1}\right)$.

Now choose $M_{2}>T_{1}$ so large that

$$
\sum_{j=1}^{T_{1}} A_{j} /\left(M_{2}+j\right)<\varepsilon_{1} .
$$

Then apply the Lemma for $N=T_{1}, M=M_{2}, \varepsilon=\varepsilon_{2}$ to obtain an integer $T_{2}>M_{2}$ and a sequence of positive numbers $A_{T_{1}+1} \geqslant A_{T_{1}+2} \geqslant \cdots>A_{T_{1}+T_{2}}$ such that

(i) $\sum_{j=1}^{M_{2}} A_{T_{1}+j} / j<\varepsilon_{2}$,

(ii) $1<\sum_{j=1}^{T_{2}} A_{T_{1}+j} /\left(T_{1}+j\right)$ but $\sum_{j=1}^{T_{2}} A_{T_{1}+j} / j<4$,

(iii) $\sum_{j=1}^{t} A_{T_{1}+t+1-j} / j<6 \varepsilon_{2}\left(1<t<T_{2}\right)$.

Now choose $M_{3}>T_{2}$ so large that

$$
\sum_{j=1}^{T_{2}} A_{T_{1}+j} /\left(M_{3}+j\right)<\varepsilon_{2}
$$

Then apply the Lemma for $N=T_{1}+T_{2}, M=M_{3}, \varepsilon=\varepsilon_{3}$ and continue inductively to obtain: two sequences of positive integers $M_{1}, M_{2}, \ldots$ and $T_{1}, T_{2}, \ldots$ satisfying

$$
M_{k}<T_{k}<M_{k+1} \quad(k \geqslant 1),
$$

and a sequence of positive numbers $A_{1}, A_{2}, \ldots$ satisfying

$$
A_{\sigma_{k}+1} \geqslant A_{\sigma_{k}+2} \geqslant \cdots \geqslant A_{\sigma_{k}+T_{k}} \quad\left(\sigma_{k} \equiv \sum_{j=0}^{k-1} T_{j} ; T_{0} \equiv 0 ; k>1\right)
$$

such that

(a) $\sum_{j=1}^{M_{k}} A_{\sigma_{k}+j} / j<\varepsilon_{k}$,

(b) $\sum_{j=1}^{T_{k}} A_{\sigma_{k}+j} /\left(\sigma_{k}+j\right)>1$ but $\sum_{j=1}^{T_{k}} A_{\sigma_{k}+j} / j<4$,

(c) $\sum_{j=1}^{T_{k}} A_{\sigma_{k}+j} /\left(M_{k+1}+j\right)<\varepsilon_{k}$,

(d) $\sum_{j=1}^{t} A_{\sigma_{k}+t+1-j} / j<6 \varepsilon_{k}\left(1<t<T_{k}\right)$.

Now we proceed to define the desired function. Inductively we obtain a sequence of mutually disjoint closed intervals $I_{n} \equiv\left[a_{n}, b_{n}\right]$ contained in $(0,1)$ such that for each index $k$ we have

$$
I_{i}<I_{\sigma_{k}+j}<I_{\sigma_{k}+j+1} \quad\left(j=1,2, \ldots, T_{k}-1 ; i>\sigma_{k+1}\right) .
$$

Then we define $f(x)=0$ if $x \notin\left(a_{n}, b_{n}\right)$ for each $n, f\left(\left(a_{n}+b_{n}\right) / 2\right)=A_{n}$, and linearly extend $f$ to the remainder of $[0,1]$.

For each index $n$ set $I_{n}^{*}=\left(a_{n},\left(a_{n}+b_{n}\right) / 2\right)$ and note that, by the first inequality in (b), we have

$$
\sum_{n=1}^{\infty} f\left(I_{n}^{*}\right) / n=\sum_{n=1}^{\infty} A_{n} / n=\sum_{k=1}^{\infty} \sum_{j=1}^{T_{k}} A_{\sigma_{k}+j} /\left(\sigma_{k}+j\right)=\infty ;
$$

that is, $f \notin H B V$. 
To show that $f \in O H B V$, it suffices to prove the finiteness of the supremum of the sums $\sum f\left(J_{n}\right) / n$ taken over all ordered sequences of intervals $J_{1}, J_{2}, \ldots$ from the collection $\left\{I_{n}^{*}\right\}_{n=1}^{\infty}$.

First suppose $J_{1}>J_{2}>\cdots$. Then it follows readily from (d) that

$$
\sum f\left(J_{n}\right) / n<6 \sum_{k=1}^{\infty} \varepsilon_{k}=6 .
$$

Now suppose $J_{1}<J_{2}<\cdots$. Choose an integer $k_{0}$ and let $J_{n_{0}+1}$, $J_{n_{0}+2}, \ldots, J_{n_{0}+t_{0}}$ be the $J_{n}$ 's that equal one of the intervals $I_{\sigma_{k_{0}}+j}^{*}(j=$ $\left.1,2, \ldots, T_{k_{0}}\right)$. Since $A_{\sigma_{k_{0}}+j}>A_{\sigma_{k_{0}}+j+1}\left(j=1,2, \ldots, T_{k_{0}}-1\right)$, we have

$$
\Sigma_{0} \equiv \sum_{j=1}^{t_{0}} f\left(J_{n_{0}+j}\right) /\left(n_{0}+j\right)<\sum_{j=1}^{t_{0}} A_{\sigma_{k_{0}}+j} / j
$$

Hence, if $t_{0}<M_{k_{0}}$, it follows from (a) that $\Sigma_{0}<\varepsilon_{k_{0}}$. Now consider the case $t_{0}>M_{k_{0}}$ : By the second inequality in (b), we have $\Sigma_{0}<4$; furthermore, if $J_{n_{0}+t_{0}+i}$ $(p<i<q)$ are the $J_{n}$ 's that equal one of the intervals $I_{\sigma_{k}+j}^{*}\left(j=1,2, \ldots, T_{k}\right)$ for a fixed $k<k_{0}$, then since $A_{\sigma_{k}+j}>A_{\sigma_{k}+j+1}\left(j=1,2, \ldots, T_{k}\right)$ we have

$$
\sum_{i=p}^{q} f\left(J_{n_{0}+t_{0}+i}\right) /\left(n_{0}+t_{0}+i\right)<\sum_{j=1}^{q-p+1} A_{\sigma_{k}+j} /\left(M_{k_{0}}+j\right)<\varepsilon_{k},
$$

where the last inequality follows from (c) and the fact that $M_{k_{0}}>M_{k+1}$ for $k<k_{0}$. Consequently,

$$
\sum f\left(J_{n}\right) / n<4+\sum_{k=1}^{\infty} \varepsilon_{k}=5
$$

and $f \in O H B V$.

The author wishes to thank A. Deacon who, via the computer, helped determine that an earlier attempt at such an example was insufficient.

\section{REFERENCES}

1. D. Waterman, On convergence of Fourier Series of functions of generalized bounded variation, Studia Math. 44 (1972), 107-117.

2.,$\Lambda$-bounded variation: recent results and unsolved problems, Real Anal. Exchange 4 (1978-79), 69-75.

Department of Mathematics, Syracuse University, Syracuse, New York 13210 